Trans

continentales
Transcontinentales

Sociétés, idéologies, système mondial

$4 \mid 2007$

Amérique latine

\title{
La démocratie locale dans les métropoles indiennes
}

Les associations de résidents à New Delhi

Local democracy in Indian metropolises: Neighbourhood associations in New Delhi

\section{Stéphanie Tawa Lama-Rewal}

\section{OpenEdition}

Journals

Édition électronique

URL : http://journals.openedition.org/transcontinentales/660

DOI : 10.4000/transcontinentales.660

ISBN : 978-2-7351-1565-5

ISSN : 1775-397X

Éditeur

Editions de la maison des sciences de l'homme

\section{Édition imprimée}

Date de publication : 1 juin 2007

Pagination : 131-144

ISBN : 978-2-200-92396-9

ISSN : 1950-1684

Référence électronique

Stéphanie Tawa Lama-Rewal, « La démocratie locale dans les métropoles indiennes »,

Transcontinentales [En ligne], 4 | 2007, document 8, mis en ligne le 28 avril 2011, consulté le 08

septembre 2020. URL : http://journals.openedition.org/transcontinentales/660 ; DOI : https://doi.org/ $10.4000 /$ transcontinentales.660 


\title{
La démocratie locale dans les métropoles indiennes
}

\author{
Les associations de résidents à New Delhi
}

\author{
Stéphanie Tawa Lama-Rewal
}

La politique de décentralisation adoptée par le Parlement indien en décembre 1992 est certainement l'une des principales réformes politiques des années 1990. Comme dans d'autres pays, les avocats de la décentralisation en firent valoir deux principaux résultats escomptés : une administration plus efficace, car tenant compte des particularités locales; et une démocratie plus solide, du fait de l'élargissement de «l'entonnoir de la représentation ${ }^{1}$ » et de la proximité accrue entre électeurs et élus. Dans le cadre de l'Union indienne, il s'agissait aussi de renforcer le fédéralisme en conférant au troisième niveau, celui de la vie politique locale, une légitimité et des moyens nouveaux (voir encadré page suivante).

La politique de décentralisation fut d'abord portée par Rajiv Gandhi (Premier ministre de 1984 à 1989), pour qui elle faisait partie d'une entreprise de modernisation de l'Etat - tout en ayant des avantages électoraux que ses opposants dénoncèrent immédiatement ${ }^{2}$. De fait, les discussions relatives à cette politique furent particulièrement animées, et les deux amendements, l'un concernant l'Inde rurale, c'est-à-dire les conseils de village, de cantons et de districts, l'autre concernant l'Inde urbaine, c'est-à-dire les municipalités, furent repoussés lors de leur première introduction en 1989, mais ils furent adoptés en 1992 sous le gouvernement congressiste de Narasimha Rao. Puisque les élections locales relèvent de la compétence des États, ceux-ci disposèrent alors d'un délai d'un an pour adopter une législation

1 - Cette expression de Rajiv Gandhi est rapportée par K. C. Sivaramakrishnan, un haut fonctionnaire étroitement associé au processus d'élaboration de cette politique à la fin des années 1980. K. C. Sivaramakrishnan, Power to the People? The Politics and Progress of Decentralisation, Delhi, Konark Publishers, 2000, p. 17.

2 - Il s'agissait aussi, pour le parti du Congrès (I) en perte de vitesse, d'aller mobiliser les électeurs à la base, et si possible de contourner les gouvernements des États, de plus en plus nombreux à quitter le giron congressiste. 
conforme aux $73^{e}$ et $74^{e}$ amendements à la Constitution. C'est seulement à partir de 1994 que des élections locales dans le cadre de la nouvelle politique de décentralisation purent avoir lieu, ce qui fut fait progressivement.

\section{LA FAIBLESSE HISTORIQUE DU GOUVERNEMENT LOCAL EN INDE}

L'importance du gouvernement local fit l'objet d'un débat, lors de la naissance de I'Inde indépendante, entre Gandhi et Ambedkar - leader intouchable qui présida le comité chargé de rédiger la Constitution. À la vision idyllique du Mahatma, qui concevait les villages comme autant de petites démocraties, reprenant ainsi à son compte le mythe des «petites républiques» popularisé par les orientalistes, Ambedkar opposa, au cours d'un discours souvent cité, la réalité sordide de I'Inde rurale, pour justifier son refus d'inclure dans la Constitution une clause relative aux panchayats [conseils] de village :

"Je tiens que ces républiques villageoises ont été la ruine de I'Inde. Je suis donc surpris que ceux qui condamnent le provincialisme et le communalisme s'avancent comme les champions du village. Qu'est-ce que le village sinon le cloaque du localisme, le repaire de l'ignorance, de l'étroitesse d'esprit et du communalisme?» (Constituent Assembly Debates, vol. VII, p. 39)

La Constitution indienne ne comportera finalement aucune clause relative au gouvernement local, sinon I'article 40 (relevant des «Principes directeurs », donc ayant valeur de recommandation seulement) selon lequel «chaque État prendra les mesures adéquates pour organiser des panchayats de village, et les investira des pouvoirs et de l'autorité nécessaires pour leur permettre de fonctionner comme des unités d'auto-gouvernement». Le concept de Panchayati Raj [gouvernement des panchayats] est introduit en 1957 par le Comité Balwantrai Mehta, chargé d'évaluer les raisons de l'inefficacité des programmes de développement locaux mis en place après I'Indépendance. L'objectif est alors d'amener la population locale à participer plus activement au développement. Le Comité recommande un nouveau dispositif, reposant sur une structure pyramidale de conseils élus à deux ou trois niveaux, selon les États. En 1959 tous les États sont dotés d'une loi relative aux panchayats $^{1}$. Mais vingt ans après le Comité Balwantrai Mehta, le gouvernement local paraît à nouveau inopérant, la "première génération de panchayats» se caractérisant par un déclin continu, "sauf dans les États du Gujarat et du Maharashtra ${ }^{2}$ ». Un nouveau Comité, sous la présidence d'Asoka Mehta, nommé en 1977, émet plusieurs recommandations pour remédier à ce qui apparaît comme un déclin général des institutions locales. Le Comité recommande de redéfinir les panchayat pour en faire des institutions politiques plutôt que des agences de mise en œuvre des programmes de développement. II suggère notamment que les partis politiques participent aux élections locales, ce qui n'était pas le cas jusqu'alors. Une deuxième génération de panchayats apparaît alors dans les États du Bengale occidental, de l'Andhra Pradesh, du Karnataka et du Kérala, qui deviennent des États pionniers de la décentralisation telle qu'elle sera définie dans les $73^{\mathrm{e}}$ et $74^{\mathrm{e}}$ amendements à la Constitution adoptés en 1992.

1-K. Sharma, "Transformative Politics: Dimensions of Women's Participation in Panchayati Raj», Indian Journal of Gender Studies, vol. 5, $n^{\circ} 1$, 1998, p. 30.

2 - G. Kumar, Local Democracy in India. Interpreting Decentralization, Delhi, Sage, 2006, p. 20. 
Cet article, centré sur les métropoles, vise à mettre en perspective le renouvellement démocratique produit par la décentralisation avec le développement contemporain de pratiques participatives, investies par les associations de résidents, pour souligner les rapports (de classe) entre les dimensions représentative et participative de la démocratie locale dans l'Inde urbaine.

\section{La politique de décentralisation : un bilan mitigé}

Douze après les débuts de la mise en œuvre des $73^{e}$ et $74^{e}$ amendements, le bilan est très mitigé. Sauf exceptions (le Kérala et, dans une moindre mesure, le Karnataka et le Bengale occidental), la réticence des États à respecter l'esprit des deux amendements en transférant fonctions, finances et fonctionnaires aux niveaux inférieurs apparaît dans les législations adoptées comme dans leur mise en ouvre. Les deux acquis majeurs de la politique de décentralisation sont en fait les mesures imposées par les deux amendements. Il s'agit, premièrement, de la régularité des élections locales : celles-ci doivent avoir lieu tous les cinq ans, sous le contrôle des commissions électorales des États, et le délai maximum entre la dissolution d'une assemblée locale et de nouvelles élections est de six mois (alors que l'État du Tamil Nadu, par exemple, avait pu suspendre les élections municipales à Madras pendant vingt-deux ans). Il s'agit, deuxièmement, des quotas pour les castes et les tribus répertoriées (en proportion de leur poids démographique local) et pour les femmes (un tiers des sièges) ${ }^{3}$. Ces deux mesures aboutissent ensemble à un véritable renouvellement du personnel politique local, qui suscite parfois de fortes résistances.

\section{Les limites au pouvoir des élus locaux}

La mise en œuvre de la politique de décentralisation en général, et des quotas féminins en particulier, a fait l'objet d'un grand nombre d'études qui se sont consacrées pour l'essentiel à l'Inde rurale, où vit plus de $70 \%$ de la population indienne, et où leur potentiel émancipatoire paraissait particulièrement fort. Une étude récente de la mise en œuvre de ces quotas féminins dans les municipalités de quatre mégapoles indiennes - Delhi, Mumbai (Bombay), Chennai (Madras) et Kolkata (Calcutta $)^{4}$ - montre en fait que dans les mégapoles, comme dans le monde rural, l'impact des quotas varie fortement en fonction des régions. Dans les régions, ou les villes, caractérisées par une vitalité relativement ancienne de la vie politique locale et par la domination de partis politiques disposant d'une structure organisationnelle dense, comme Kolkata et dans une moindre mesure Mumbai, l'impact des quotas sur la participation politique des femmes est fort : entre la première et la deuxième élection sous le $74^{\mathrm{e}}$ amendement, le nombre de femmes candidates à des sièges non réservés a augmenté substantiellement, de même que le nombre de femmes élues; de plus en plus de femmes occupent des positions décisives

3 - Ces derniers quotas ont frappé les imaginations, dans la mesure où les femmes, contrairement aux deux autres catégories mentionnées, ne disposaient jusqu'alors d'aucun quota électoral aux niveaux national et régional.

4 - Archana Ghosh et Stéphanie Tawa Lama-Rewal, Democratization in Progress : Women and Local Politics in Urban India, Delhi, Tulika, 2005. 
dans les divers comités municipaux; et la participation des femmes élues dans les débats municipaux est proportionnelle à leur présence dans le conseil municipal. À l'inverse, à Delhi comme à Chennai, très peu de femmes sont candidates (et encore moins élues) à des sièges non réservés, et les élues ne disposent pas des ressources politiques nécessaires pour exercer pleinement leurs nouvelles fonctions; ou du moins, pour les exercer aussi pleinement que leurs collègues masculins.

Car cette enquête souligne que plusieurs facteurs institutionnels font obstacle à la capacité d'agir des élus des deux sexes. Tout d'abord, l'existence maintenue des nombreuses agences étatiques et para-étatiques en charge de la gestion des services essentiels réduit les municipalités à une fonction d'entretien des infrastructures urbaines. Ensuite, c'est le régime municipal dit "système du commissaire» qui domine les municipalités, à l'exception du Bengale occidental (et plus récemment du Madhya Pradesh). Dans ce régime, le Commissaire municipal, un haut fonctionnaire nommé par le gouvernement de l'État qui dirige l'aile exécutive de la municipalité, dispose de pouvoirs tels qu'il éclipse totalement le maire, réduit à un rôle largement honorifique.

\section{Le déficit de proximité du gouvernement local}

Les circonscriptions électorales municipales sont immenses en termes de population (notamment à Delhi, où elles comptent en moyenne 100000 habitants), et la plupart des États n'ont pas créé le "deuxième niveau» de décentralisation préconisé par les amendements, un niveau inférieur, situé à une échelle suffisamment restreinte pour favoriser la participation des habitants à la gestion des affaires locales. Les ward committees, censés être des lieux de rencontre entre l'élu local, l'administration en charge du quartier, et les habitants représentés par la société civile organisée, sont largement restés lettre morte.

Enfin le taux de participation électorale, toujours plus bas pour les élections municipales que pour les élections à l'Assemblée de l'État et au Parlement ${ }^{5}$, laisse à penser que les électeurs sont relativement indifférents à l'activité des municipalités ${ }^{6}$, et suggère que la mise en œuvre de la politique de décentralisation n'a pas produit le renouvellement démocratique escompté.

Dans de nombreuses métropoles indiennes, on observe pourtant une implication croissante des habitants dans la gestion des affaires locales, principalement à travers des associations de résidents, les Resident Welfare Associations, de plus en plus nombreuses

5 - Le taux de participation était de $65 \%$ à Kolkata (où la participation électorale est toujours la plus forte de l'Inde) en 2000, 35 \% à Chennai en 2001 (mais la violence qui a marqué ces élections joue un rôle dans ce chiffre particulièrement bas), 43 \% à Mumbai et 42 \% à Delhi en 2002.

6 - Il faut également noter que le taux de participation aux élections nationales est, depuis 1991, systématiquement plus bas dans les circonscriptions urbaines (53,1\% en 2004) que dans les circonscriptions rurales (58,9 \% en 2004) (Christophe Jaffrelot, " "Why should we vote?" The Declining Electoral Participation of the Urban Middle Class in India», communication présentée lors du colloque Consumerism and the Emerging Middle Class: Comparative Perspectives from India and China, Delhi, 7-9 novembre 2005). 
(voir encadré page suivante) et de plus en plus visibles, qui se réclament de la démocratie participative et se présentent comme les porte-parole de citadins citoyens.

Comment qualifier cette émergence des associations de résidents sur la scène publique urbaine, comment la situer dans l'analyse de la démocratie locale? Les éléments de réponse proposés ici sont fondés sur l'étude de ces associations à Delhi, et convergent sur l'essentiel avec les travaux sur le même sujet de Janaki Nair à Bangalore ${ }^{7}$, Karen Coelho $^{8}$, John Harriss ${ }^{9}$ et Pushpa Arabindoo ${ }^{10}$ à Chennai, Sharit Bhowmik ${ }^{11}$ et Marie-Hélène Zérah ${ }^{12}$ à Mumbai, et Loraine Kennedy à Hyderabad ${ }^{13}$.

Les associations de résidents, hérauts improbables de la démocratie participative

Les associations de résidents existent au moins depuis les années 1970 à Delhi. Ce sont des associations à but non lucratif, dont les obligations, pour être enregistrées légalement, se limitent à avoir sept membres fondateurs, se réunir régulièrement, et se doter de statuts dont le contenu est laissé à la discrétion de l'association. Ces associations ont en général pour raison d'être des préoccupations relatives à l'état des infrastructures et des services de base du quartier : routes, parc, approvisionnement en eau et en électricité, collecte des ordures ménagères ${ }^{14}$. Elles s'attachent aussi à favoriser une certaine convivialité locale ${ }^{15}$, en organisant la célébration de certains festivals (laïcs ou hindous), voire en mettant en place un service d'entraide informel ${ }^{16}$.

7 - Janaki Nair, «"Social Municipalism” and the New Metropolis», in Mary E. John, Praveen Kumar Jha et Surinder S. Jodhka (éds), Contested Transformations. Changing Economies and Identities in Contemporary India, Delhi, Tulika, 2006, p. 125-146.

8 - Karen Coelho, "Civil and Uncivil Mobilizations. A Report of the Preliminary Mapping Study on Collective Action Related to Service Delivery in Chennai, South India», September 2005.

9 - John Harriss, "Middle Class Activism and Poor People's Politics: An Exploration of Civil Society in Chennai ", London School of Economics and Political Science, Working Paper Series, $\mathrm{n}^{\circ} 05$ 72, 2005, $37 \mathrm{p}$.

10 - Pushpa Arabindoo, «Residents Associations: An Alternative Civil Society?», Ph.thesis, London School of Economics and Political Science, soutenance prévue en 2007.

11 - Sharit Bhowmik, "The Politics of Urban Space in Mumbai. "Citizens" versus the Urban Poor", in Mary E. John, Praveen Kumar Jha et Surinder S. Jodhka (éds), op. cit., 2006, p. 147-162.

12 - Marie-Hélène Zérah, «Emerging Links between Urban Management, Collective Action and (re)Mobilization of the Middle Class in Mumbai - Framing Hypothesis and Preliminary Findings », communication présentée dans le cadre du séminaire mensuel du Centre de sciences humaines de New Delhi, Forms, Objects and Stakes of Political Mobilizations in Contemporary India, le 26 juillet 2006 .

13 - Loraine Kennedy, «Decentralisation and Urban Governance in Hyderabad. Assessing the Role of Different Actors in the City", communication présentée lors du Workshop on Urban Actors, Policies and Governance in Hyderabad, Administrative Staff College of India, Hyderabad, 20 septembre 2005, 20 p.

14 - À Mumbai, beaucoup se sont formées à la faveur du «Advanced Locality Management Scheme», centré sur la question de la collecte des ordures ménagères (Marie-Hélène Zérah, op. cit.).

15 - Un président d'une association de résidents me dit ainsi avec fierté : «Nous avons transformé tout le lotissement en une vraie communauté.» (Entretien avec DK, octobre 2006)

16 - Plus récemment, et à la suite de leur mobilisation dans ces domaines, les associations de résidents de Delhi offrent une sorte de service de médiation entre les résidents et les autorités locales, en mettant en place une permanence au moment où les habitants doivent calculer et payer leur impôt foncier, en collectant les factures d'eau et d'électricité pour les apporter aux agences concernées, etc. 


\section{COMBIEN D'ASSOCIATIONS DE RÉSIDENTS DANS LA VILLE?}

À Delhi, il est très difficile de connaître le nombre exact des associations de résidents, d'une part parce qu'elle peuvent fonctionner sans être enregistrées par le Registrar of Societies (qui exige pour cela la signature de sept membres fondateurs, le paiement de frais d'enregistrement modestes, une adresse permanente, une liste des objectifs, un règlement, et un rapport annuel des activités); d'autre part, parce que les informations fournies oralement par le Registrar of Societies, selon qui Delhi compterait plusieurs milliers d'associations de résidents, sont en complète contradiction avec les données fournies par son site Internet (qui offre une liste de 870 associations).

L'autre source de données est la cellule en charge du plan Bhagidari, qui ne peut donner que des chiffres incomplets puisque ce plan inclut seulement les associations pouvant faire la preuve d'une certaine ancienneté, d'élections annuelles, et étant enregistrées. La liste, non datée, fournie par cette cellule, fait état de 1096 associations de résidents, mais le chef de la cellule déclare qu'en octobre 2006, le plan Bhagidari compte 1800 associations. Si I'on estime que la réalité se trouve entre ces deux chiffres (soit 1448 associations enregistrées dans le cadre du plan Bhagidari), et en tenant compte des associations non enregistrées, on obtient une estimation large de 2000 associations de résidents environ. Chaque association représente environ 200 ménages, et si l'on estime que chaque ménage compte cinq personnes, on peut donc penser que les associations de résidents parlent au nom de deux millions de personnes (sur une population totale de 12,8 millions d'habitants, d'après le recensement de 2001). À titre de comparaison, à Bangalore, une ville de 5,7 millions d'habitants, le nombre d'associations de résidents en 2002 serait de $150^{1}$.

1 - Janaki Nair, "Social Municipalism" and the New Metropolis», in Mary E. John, Praveen Kumar Jha et Surinder S. Jodhka (éds), Contested Transformations. Changing Economies and Identities in Contemporary India, Delhi, Tulika, 2006, p. 125146, p. 141.

L'étude approfondie d'une quinzaine de ces associations, dans différents quartiers de la ville, met en lumière deux caractéristiques qui pourraient a priori les disqualifier comme sites privilégiés de la démocratie participative. Premièrement, le fonctionnement de ces associations est en général non démocratique. Leur statut les laisse libres de décider de la fréquence des élections de leur bureau, de la possibilité pour les membres du bureau de renouveler leur mandat, d'un quorum éventuel pour les assemblées générales. En pratique, les associations résultent de l'autosélection d'un petit groupe, qui monopolise souvent pour de longues périodes les différentes positions du bureau en les occupant à tour de rôle. La plupart organisent des élections, mais il arrive souvent qu'un seul candidat se manifeste pour un poste, et que les élections aient lieu à main levée. Le fonctionnement de l'association repose largement sur les réunions mensuelles du bureau, qui préfère la persuasion au vote pour trancher les conflits éventuels.

Deuxièmement, ces associations se caractérisent par une relative homogénéité sociologique, pour des raisons à la fois historiques et pratiques. Historiquement, elles sont liées au processus d'occupation des lotissements vendus et/ou construits 
par la Delhi Development Authority, institution para-étatique créée en 1957 et chargée de la préparation puis de l'application du plan d'urbanisme de la capitale. La procédure d'attribution de nombreux lotissements supposait en effet que se forment des sociétés coopératives, les cooperative (group) housing societies, qui postulent en tant que telles à l'attribution d'un terrain à bâtir ou d'un immeuble déjà construit, l'attribution se faisant ensuite par tirage au sort parmi la liste des candidats. Cette procédure concerne surtout les immeubles collectifs, mais on la trouve aussi dans des lotissements pavillonnaires. Or la formation des sociétés coopératives procède selon un principe de cooptation, explicite dans les statuts.

Quelques décennies après la formation de la société coopérative, les résidents du lotissement ne se confondent pas avec les membres originels de celle-ci, et c'est l'une des raisons de la formation des associations de résidents, qui entendent faire valoir le droit à la parole des résidents effectifs - nouveaux propriétaires, héritiers, ou, quoique moins souvent, locataires. Mais une homogénéité certaine, en termes de catégorie socioprofessionnelle, demeure ${ }^{17}$.

Ensuite, les associations de résidents sont largement confinées aux lotissements planifiés, ou régularisés; elles ne concernent ni les lotissements illégaux (les unauthorized colonies), ni les bidonvilles (habitat illégal et précaire), qui concerneraient chacun environ trois millions d'habitants ${ }^{18}$, soit près de la moitié, en tout, de la population de Delhi.

À cela s'ajoute le fait que la grande majorité des membres actifs des associations de résidents sont, comme en témoignent leurs annuaires, des hommes de plus de 60 ans $^{19}$, parmi lesquels de nombreux anciens bureaucrates ${ }^{20}$, et quelques membres des professions libérales (notamment des avocats). À Bangalore, plus nombreuses sont les femmes au foyer parmi les membres actifs des associations de résidents ${ }^{21}$, et à Mumbai et Chennai, les membres des professions libérales ${ }^{22}$.

On peut donc dire, pour résumer, que les associations de résidents, loin de représenter la diversité de la population urbaine, concernent essentiellement la population ayant accédé à la propriété (depuis plus ou moins longtemps); et qu'elles ont pour porte-parole une élite dont le profil sociologique varie en fonction des villes.

17 - Dans son enquête sur les associations de résidents à Chennai, Pushpa Arabindoo montre que celles-ci sont très présentes dans les immeubles nouvellement construits, et occupés par une classe aisée.

18 - Chiffres cités par Véronique Dupont et Usha Ramanathan, «Du traitement des slums à Delhi», Purushartha, n²6, "La ville en Asie du Sud», coordonné par V. Dupont et D.G. Heuzé, Paris, EHESS, 2007.

19 - Les retraités disposent en abondance de temps, ressource essentielle à ce type d'activité.

20 - Ce fait reflète l'importance, propre à Delhi qui est la ville de l'administration centrale, de la population active employée dans le secteur public : 28 \% en 1981 (Véronique Dupont, "Pratiques résidentielles dans le parc de logements publics d'une grande métropole indienne: l'exemple des DDA flats de Delhi», Autrepart, $\mathrm{n}^{\circ}$ 25, «Dynamiques résidentielles dans les villes du Sud : positions sociales en recomposition", coordonné par M. Bertrand, 3/2003, p. 21-36).

21 - Janaki Nair, op. cit., p. 130

22 - Marie-Hélène Zérah, op. cit. ; John Harriss, op. cit., p. 18. 
Ces caractéristiques sociologiques constituent-t-elles une ressource ou au contraire un obstacle à l'affirmation des associations de résidents sur la scène publique? Plus généralement, comment peut-on expliquer leur promotion, au cours des dix dernières années, au statut d'acteur légitime de la gouvernance ${ }^{23}$ urbaine? Quatre types de facteurs explicatifs apparaissent, qui ne sont pas exclusifs les uns des autres : le rôle de quelques leaders politiques férus de «bonne gouvernance»; la capacité des associations à défendre certains intérêts; leur discours auto-légitimant; et l'attention que leur accordent la presse et les juges.

\section{Le rôle des chefs de gouvernement des États fédérés}

Tout d'abord, les études disponibles soulignent le rôle décisif de quelques chefs de gouvernement au niveau des États se réclamant de la nouvelle gestion publique et de la notion de partenariat entre le public et le privé (qui peut représenter soit les entreprises, soit le secteur associatif). À Bangalore, le gouvernement régional et les entreprises privées ont joué de concert : en 1995, le chef du gouvernement de l'État du Karnataka, Deve Gowda, lance le Swabhimana Forum, défini comme « une initiative associant citoyens et collectivités locales pour un Bangalore plus propre, plus vert et plus sûr", et qui invite les entreprises de la ville à participer à son entretien en finançant la restauration des parcs, l'éclairage public, etc. ${ }^{24}$.

À Delhi, c'est Sheila Dixit, chef du gouvernement élue en 1998 et réélue en 2003, qui semble à première vue constituer la principale ressource de la légitimité nouvelle des associations de résidents. Elle fait grand usage, elle aussi, de la rhétorique internationale de la "bonne gouvernance» ${ }^{25}$, promettant aux habitants de la capitale de meilleures infrastructures (au premier rang desquelles les transports - réseau routier et métro), mais aussi plus de transparence, plus d'imputabilité, et plus de participation. De fait, Delhi est le seul État indien à s'être doté d'une «Commission pour les réformes administratives» permanente ${ }^{26}$; et son gouvernement a été l'un des premiers, en 2001, à adopter le droit à l'information, une législation potentiellement très "capacitante» (empowering), renforcée ici par la

23 - La notion de gouvernance, qui met l'accent d'une part sur la dimension horizontale, d'autre part sur le caractère informel des relations entre acteurs impliquées dans la gestion des affaires urbaines, est indispensable à l'analyse de l'impact de la mobilisation des associations de résidents sur la démocratie locale. Je souscris donc ici à la définition offerte par les Nations unies-Habitat : «La gouvernance urbaine est la somme des nombreux moyens par lesquels les individus et les institutions, publiques et privées, planifient et gèrent les affaires communes de la ville. C'est un processus continu au travers duquel les intérêts conflictuels ou divergents peuvent être accommodés et des actions entreprises en coopération. Cela inclut les institutions formelles aussi bien que les arrangements informels et le capital social des citadins. " (http://www.unhabitat.org/campaigns/governance/principles.asp)

24 - Quatre ans plus tard, le gouvernement congressiste de S. M. Krishna constitue la Bangalore Agenda Task Force (BATF), un comité chargé de superviser le développement de la ville, dont le vocabulaire (faisant grands cas de la notion de stakeholders) et les méthodes (des fiches d'évaluation sur la qualité des services publics) sont explicitement inspirés par l'idéologie de la "bonne gouvernance» (Janaki Nair, op. cit.). À Mumbai, l'ONG Bombay First, soutenue par le secteur privé (Sharit Bhowmik, op. cit., p. 155), joue un rôle similaire à celui du Swabhimana Forum.

25 - Sheila Dixit met régulièrement en valeur la reconnaissance internationale que lui vaut le Bhagidari scheme à travers plusieurs distinctions octroyées par les Nations unies et la Banque mondiale.

26 - Amita Singh (éd.), Administrative Reforms. Towards Sustainable Practices, Delhi, Sage, 2005, p. 44. 
mise en place d'une autorité d'appel, la Commission des plaintes publiques (Public Grievance Commission).

Mais le programme gouvernemental qui incarne le mieux l'engagement de Sheila Dixit dans la bonne gouvernance, c'est le plan Bhagidari (littéralement, le "partenariat»), lancé en 2000, et dont l'objectif affiché est de faire des citoyens des partenaires actifs de la gestion des affaires urbaines. Une cellule a été créée dans le cabinet de Sheila Dixit, consacrée au plan Bhagidari, qui procède en mobilisant les associations de résidents, invitées à s'inscrire et à devenir ainsi membres de «l'équipe Delhi» dont $\mathrm{M}^{\mathrm{me}}$ Dixit se pose en capitaine. La construction du partenariat entre citoyens et autorités locales (c'est-à-dire la principale municipalité de Delhi - la Delhi Municipal Corporation -, mais aussi le gouvernement de Delhi et les agences para-étatiques en charge de différents services ${ }^{27}$ ) passe d'abord par des rencontres, au cours d'ateliers thématiques de quelques jours, entre représentants des associations de résidents et représentants des diverses administrations. L'initiative Bhagidari établit progressivement une sorte d'autorité des associations de résidents en matière d'exécution des travaux publics dans leur quartier, d'abord en leur confiant les coordonnées personnelles des administrateurs responsables; puis en obligeant certains employés municipaux à leur rendre des comptes; enfin en conférant aux résidents une fonction consultative limitée en les associant à certaines décisions en matière d'aménagement local au niveau décentralisé du district ${ }^{28}$, ou bien en les invitant à participer à des rencontres thématiques, par exemple sur les programmes gouvernementaux et municipaux d'assistance aux personnes âgées.

Le plan Bhagidari est disqualifié par les critiques de Sheila Dixit, comme une manœuvre électoraliste destinée essentiellement à construire un réseau de clients pour l'aider à surmonter son statut originel d'outsider dans la vie politique de la capitale et l'opposition qu'elle doit affronter dans son propre parti. Il est vrai que ce programme fait l'objet d'une communication très importante, qui met invariablement l'accent sur l'engagement personnel de la Ministre en chef. Il est vrai également que Sheila Dixit semble jouir d'une grande popularité parmi les "bhagidars". Mais plus généralement, le prestige social que confère l'appartenance à «l'équipe Delhi » et les multiples occasions qu'offrent le plan de parler d'égal à égal avec un fonctionnaire haut placé dans la hiérarchie de la municipalité ou du gouvernement constituent un grand facteur de son attractivité ${ }^{29}$.

27 - Du fait de son statut historique et politique particulier, on peut considérer que le processus de décentralisation démocratique à Delhi date de l'adoption, en 1991, du 69e amendement à la Constitution, faisant de Delhi un «territoire de la Capitale nationale» (National Capital Territory), c'est-à-dire un quasi-État, doté d'une assemblée législative, d'un conseil des ministres et d'un Ministre en chef. Contrairement aux autres États toutefois, la police et l'urbanisme ne dépendent pas de «l'État» mais du Centre. La gestion des affaires locales à Delhi relève en fait d'une multitude d'autorités (le Centre, le territoire de la Capitale nationale, les trois municipalités, mais aussi une série d'agences paraétatiques chargées de l'eau, de l'électricité, du métro) dont les compétences se chevauchent parfois.

28 - Le quasi-État de Delhi compte neuf districts.

29 - «Notre député à l'Assemblée legislative nous témoigne un grand respect. » «Les ministres nous témoignent de la consideration. Je peux rencontrer la Ministre en chef n'importe quand.» (Entretiens avec DK et OPS, octobre 2006) 
Quelles que soient les motivations premières de la Ministre en chef, il semble indubitable aujourd'hui que le plan Bhagidari a joué un rôle majeur dans la promotion des associations de résidents au rang d'acteurs légitimes de la gouvernance urbaine, mais la série de conflits opposant ces associations aux autorités locales au cours des deux dernières années montre que les associations ont interprété leur mandat au-delà des intentions du plan.

\section{Une défense efficace des intérêts des consommateurs}

Entre 2004 et 2006, les associations de résidents se sont constituées en forums de défense des intérêts des consommateurs. Se réunissant en divers fronts et fédérations ${ }^{30}$, dont deux prétendent représenter des associations de toute la ville, elles ont obtenu la régularisation des opérateurs du câble, la révision de la méthode de calcul de l'impôt foncier collecté par la municipalité et la prise en charge, par le gouvernement de Delhi, de l'augmentation de $10 \%$ du prix de l'électricité à la suite de la privatisation de sa distribution. Les associations de résidents ont également participé aux protestations contre la privatisation de la distribution de l'eau, qui a abouti, en 2005, au retrait de la Banque mondiale d'un projet important. Enfin, en 2006, les associations de résidents se sont trouvées au cœur du conflit violent suscité par la mise en œuvre du schéma directeur d'urbanisme de la ville.

Parmi ces cinq conflits, les trois derniers ont un potentiel déstabilisateur pour le gouvernement de Delhi, comme pour la municipalité, tous deux dominés par le parti du Congrès. Même s'il ne fait aucun doute que le principal parti d'opposition, le Bharatiya Janata Party, intervient en soutenant certaines fédérations d'associations de résidents, ces associations semblent avoir acquis un poids imprévu dans les relations entre les acteurs politiques, administratifs et économiques de la gouvernance urbaine. Alors que cette dernière est de plus en plus marquée par l'idéologie de la nouvelle gestion publique, cette capacité à procurer des bénéfices tangibles aux résidents renforce la légitimité des associations de résidents comme instances de représentation ${ }^{31}$.

\section{Un discours auto-légitimant}

Le troisième facteur explicatif de l'importance nouvelle de ces associations tient justement à leur discours, qui évite les notions de groupe d'intérêts, et plus encore de lobby. Il relève d'un usage stratégique des mots, qui permet à une minorité active de se poser en représentante de la majorité. Les déclarations à la presse (que pratiquent

30 - Il s'agit là d'un phénomène nouveau, et particulier à Delhi (les travaux de Harriss et Arabindoo sur Chennai soulignent que les associations de résidents sont rétives à l'idée de se regrouper) : alors que de petites fédérations existaient sur la base de la contiguïé géographique des lotissements, pour parler d'une voix commune face aux autorités sur des problèmes communs (par exemple, une route traversant plusieurs lotissements), le Delhi Joint Front of Resident Welfare Associations, créé en 2002, comme le United Residents Joint Association, créé en 2004, rassemblent chacun plusieurs centaines d'associations, réparties dans plusieurs zones de la ville, et parlent au nom des "résidents de Delhi ».

31 - «Bhagidari signifie shareholder.» (Entretien avec D\&B, octobre 2006) 
beaucoup ces associations) favorisent d'une part le contraste entre les petits ("la classe moyenne», "les gens ordinaires», «les consommateurs affligés», "les gens de Delhi») et les gros ou les nombreux («les politiciens et leurs banques de votes», "les grands propriétaires fonciers dans les villages urbains", "le gouvernement», "les compagnies électriques privées»); d'autre part l'opposition entre les honnêtes gens ( "les résidents qui respectent la loi», «le contribuable honnête», «le consommateur honnête», "chaque membre honnête de la classe moyenne») et les autres («les industries non autorisées", «les bureaucrates corrompus», "la mafia immobilière», «ceux qui violent la loi », «ceux qui empiètent sur des terrains publics») ${ }^{32}$. Parmi le vocabulaire privilégié par ces déclarations à la presse, les notions de «citoyens » et de «classe moyenne» sont plus particulièrement récurrentes.

Or, comme l'a montré Satish Deshpande, la notion de classe moyenne, aussi vague qu'omniprésente, est particulièrement fallacieuse. Le mot, d'une part, évoque le milieu de la hiérarchie socioéconomique, mais aussi la majorité. D'autre part, le discours sur la classe moyenne associe à cette notion des images de consommateurs profitant des biens que la libéralisation économique met à leur portée, travaillant dans le secteur tertiaire et ayant une certaine connaissance de l'anglais. Or, en s'appuyant sur une série d'enquêtes statistiques, à l'échelle nationale, sur les dépenses de consommation, Deshpande montre que les catégories situées au milieu de la hiérarchie socioéconomique sont très loin de correspondre à cette image ${ }^{33}$, et que même en adoptant une définition large de la classe moyenne, c'est-à-dire en situant sa limite inférieure au niveau de consommation où le mode de transport principal des ménages est un deux-roues motorisé, on arrive à une estimation de la taille de la classe moyenne à $15 \%$, au maximum, de la population indienne ${ }^{34}$; le pluriel mais aussi les guillemets sont donc de rigueur dans l'emploi de cette notion. Même si l'on considère que ces «classes moyennes» sont plus importantes dans les villes que dans les campagnes, et a fortiori dans une ville comme Delhi, il est certain que les «classes moyennes» ne peuvent prétendre constituer la majorité.

La deuxième notion privilégiée par les associations de résidents est celle de "citoyens». Les associations soulignent leur vertu civique pour mieux dénoncer la corruption, l'infraction à la loi des autres. Elles s'approprient la rhétorique du plan Bhagidari, qui les désigne comme "groupes de citoyens», conférant ainsi une dimension politique, au sens noble, aux relations entre administrateurs et

32 - Source : pages «Delhi» du Hindu, 2004-2006. On peut faire deux remarques sur le choix de ce vocabulaire : d'une part les associations de résidents ne font jamais valoir qu'ils sont (aussi) des électeurs, ce qui manifeste leur défiance du processus électoral; d'autre part ils ne font allusion aux pauvres que sous des formes euphémisées par la dépersonnalisation ( «les quartiers de jhuggi», c'està-dire les bidonvilles), ou en les désignant par leur infraction à la loi («les violeurs de la législation», "ceux qui empiètent sur l'espace public»).

33 - "[...] ce que l'on appelle populairement la "classe moyenne" indienne n'est en réalité pas au milieu, mais en haut de l'échelle des revenus [...] seulement $5 \%$ de la population indienne dépense plus de... Rs 1925 [38 euros] par personne et par mois dans l'Inde urbaine - les $95 \%$ restant dépensent moins.» (Satish Deshpande, "Mapping the "Middle". Issues in Analysis of the "Non-Poor" Classes in India», in Mary E. John, Praveen Kumar Jha et Surinder S. Jodhka (éds), Contested Transformations. Changing Economies and Identities in Contemporary India, Delhi, Tulika, 2006, p. 215-236, p. 218-220).

34 - Satish Deshpande, op. cit., p. 222. 
administrés $^{35}$. La notion de citoyenneté souligne la légitimité des associations de résidents comme interlocuteurs de l'État, en évoquant un ensemble de droits et de devoirs réciproques. Or l'essentiel du discours des associations de résidents procède justement en deux temps: d'abord, rappeler les devoirs remplis (les services assurés, les factures et impôts dûment payés); puis souligner les droits bafoués (droit à un approvisionnement continu en eau et en électricité, à un environnement salubre, etc.).

\section{L'attention des médias et des juges}

La capacité des associations de résidents à faire entendre ce discours nous amène au quatrième facteur explicatif : l'attention, sinon le soutien, que leur apportent ces deux institutions essentielles de la démocratie que sont les médias et le pouvoir judiciaire. D'une part, la presse accorde une large couverture aux faits et dits des associations de résidents, et surtout de leurs fédérations. Que les deux plus importantes de ces fédérations, à Delhi, soient dirigées par des professionnels de la communication n'est sans doute pas étranger à cet état de choses. Mais selon les organisations opposées aux associations de résidents sur la question de la démolition des bidonvilles, organisations qui s'efforcent elles aussi, mais sans succès, de promouvoir leur cause à travers les médias, la presse est clairement plus réceptive aux informations mettant en jeu des gens auxquels leurs lecteurs sont susceptibles de s'identifier. La presse, en acceptant l'auto-définition des associations de résidents comme les représentants des habitants, en les traitant comme «la voix des résidents» (selon l'expression du quotidien Hindustan Times), contribue significativement à leur légitimité.

D'autre part, la Haute Cour de Delhi comme la Cour suprême, qui sont intervenues à maintes reprises au cours des dix dernières années dans la gestion des affaires urbaines, manifestent, dans leurs déclarations, une empathie frappante avec les revendications des associations de résidents, notamment sur les questions touchant à l'environnement ${ }^{36}$. Dans leur analyse de la gestion, par le judiciaire, de la question des bidonvilles, Véronique Dupont et Usha Ramanathan ont montré que les jugements reflètent de plus en plus fréquemment un biais favorable aux "classes moyennes", et qu'une mesure telle que le litige d'intérêt public, créé à la fin des années 1970 pour défendre les droits essentiels des pauvres, est, depuis le milieu des années 1990, fréquemment utilisée par les «classes moyennes» contre les pauvres ${ }^{37}$. De fait, les fédérations d'associations de résidents font grand usage des litiges d'intérêt public; bénéficiant du service gratuit des avocats qui se trouvent

35 - Mais au-delà du plan Bhagidari, on peut remarquer que de nombreuses ONG, à Delhi et ailleurs, se présentent elles aussi comme des "groupes (de) citoyens", manifestant ainsi un rapport au politique qui contraste avec l'affirmation traditionnelle de leur apolitisme.

36 - Sur une série de controverses impliquant la qualité de l'environnement naturel, à Delhi (déplacement des industries polluantes, passage au gaz naturel compressé pour les véhicules de transport public, nettoyage de la rivière Yamuna) comme à Bangalore (préservation du parc Cubbon), les associations de résidents comme les juges privilégient les considérations écologiques au détriment de leurs conséquences sociales.

37 - Véronique Dupont et Usha Ramanathan, op. cit. 
dans leurs rangs, elles affirment ainsi, encore une fois, que la défense de leurs intérêts est celle de l'intérêt général ${ }^{38}$.

\section{Conclusion}

L'émergence des associations de résidents comme acteurs de la gouvernance urbaine dans plusieurs métropoles indiennes ${ }^{39}$ constitue en soi un facteur important de renouvellement de la démocratie locale, tant en ce qui concerne les catégories sociales mobilisées que les modalités et l'échelle de leur action collective. Les associations de résidents apparaissent comme des structures mobilisatrices qui ont su imposer le lotissement ou le quartier comme unités pertinentes dans la gestion des affaires locales. Elles souffrent certes d'un déficit démocratique - puisqu'elles reposent largement sur la cooptation, ne sont pas représentatives de l'ensemble des résidents et pratiquent le consensus plus que la délibération - mais elles sont conscientes que ce défaut constitue un risque majeur de délégitimation, comme en témoigne leur souci de tenir les minutes de leurs réunions, de faire auditer leurs comptes et d'organiser des élections de manière à la fois plus régulière et plus libre. De surcroît, ces associations remplissent une fonction importante en démocratie : celle de la surveillance des gouvernants par les gouvernés; en cela, elles contribuent indubitablement à l'augmentation de la transparence et de l'imputabilité des autorités locales.

Une des limites principales de cette participation est évidemment son caractère élitiste : les "classes moyennes» dont les associations de résidents se font les porte-parole efficaces sont en fait une élite surdotée en ressources politiques (temps, éducation, argent, familiarité avec la bureaucratie, compétences en relations publiques, compétences juridiques, etc.). Cette limite n'est pas propre à l'Inde : comme en témoignent les travaux sur le sujet, qui se multiplient au même rythme que les expériences de démocratie participative dans le monde ${ }^{40}$, les procédures participatives sont sujettes à la domination par les minorités actives qui prennent souvent la forme de la société civile organisée.

Mais le contexte indien a ceci de particulier que cette mobilisation des "classes moyennes» contraste avec leur apathie électorale : la démocratie indienne est en effet caractérisée par le fait que ce sont les plus pauvres qui votent le plus régulièrement, à rebours de la tendance générale observée ailleurs. À cet égard, la réflexion de Partha Chatterjee sur les relations entre "société civile» et "société politique» en Inde offre une grille d'interprétation des phénomènes décrits ici. Pour Chatterjee, la société politique, structurée par les partis politiques, est le site des mobilisations de ceux qui «survivent en enfreignant la loi » et revendiquent des droits non en tant qu'individus

38 - Le deuxième outil législatif de la démocratisation dont usent abondamment les associations de résidents est le droit à l'information.

39 - La mobilisation simultanée des associations de résidents dans plusieurs métropoles indiennes s'explique notamment par un échange, parfois organisé par les acteurs gouvernementaux, parfois d'initiative privée, entre les organisations ayant joué un rôle moteur. Ainsi l'ONG Janaagraha, une émanation de la Bangalore Agenda Task Force (BATF) à Bangalore, a contribué à la conceptualisation du plan Bhagidari à Delhi, que la municipalité de Mumbai tente actuellement d'adapter.

40 - Voir Marie-Hélène Bacqué, Henri Rey et Yves Sintomer (dir.), Gestion de proximité et démocratie participative. Une perspective comparative, Paris, La Découverte, 2005. 
mais en tant que "communautés», tandis que la société civile regroupe les "citoyens à proprement parler»; selon lui, "nous pourrions bien être en train d'assister à une opposition émergente entre modernité et démocratie, c'est-à-dire entre société civile et société politique» ${ }^{41}$.

La place prise par les associations de résidents à la faveur de la mise en place de procédures participatives dans la gouvernance urbaine tend en effet à marginaliser la dimension représentative de la démocratie locale, avec des conséquences tragiques pour les pauvres. Le profil sociologique des membres actifs de ces associations, leur tendance à préférer comme interlocuteurs les bureaucrates plutôt que les élus des autorités locales (pour des raisons tenant à la fois à leur proximité sociologique et à leur conception négative de la politique ${ }^{42}$ ) limitent l'impact de la politique de décentralisation, dont l'un des aspects majeurs, on l'a vu, est la démocratisation de la vie politique locale au moyen de quotas. Surtout, l'efficacité de la "prise de parole» des associations de résidents pèse d'un poids certain en faveur des privilégiés, contre les pauvres, dans un conflit comme celui qui porte sur l'occupation du sol à Delhi, et qui est arbitré par la Cour suprême.

La «société politique» n'a cependant pas dit son dernier mot. Le ministère du Développement urbain au niveau de l'Union vient de lancer une mission de consultation sur le «renouvellement urbain national», qui comporte notamment un projet de loi, dit «loi de participation communautaire» (Community participation law). Ce projet de loi propose de pallier le déficit de participation dans le cadre de la décentralisation en créant dans les villes des "assemblées de zones» (area sabha) à l'échelle d'un ou deux bureaux de vote - soit, à Delhi, des unités correspondant à 1500 à 3000 personnes, c'est-à-dire au moins trente-trois fois plus petites que les actuelles circonscriptions municipales - et dont les membres seraient nommés par l'élu de la zone, que ce soit l'élu municipal, le député au niveau de l'État ou celui au niveau du Parlement. Ce retour inattendu du principe nominatif manifeste sans aucun doute le désir des acteurs politiques locaux de contrôler une démocratie participative en passe, dans certains cas, de les marginaliser.

41 - Partha Chatterjee, «On Civil and Political Society in Post-Colonial Democracies», in Sudipta Kaviraj et Sunil Khilnani (éds), Civil Society, History and Possibilities, Cambridge, Cambridge University Press, 2003, p. 165-178, p. 178.

42 - Pour autant, les associations de résidents sont également associées à des initiatives visant à réformer la politique au sens classique, par le biais d'ONG telles que Lok Satta et PUCAAR (People's Union for Civic Action and Rights) à Hyderabad, AGNI (Action for Good Governance and Networking in India) à Mumbai ou People's Action à Delhi, qui ont en commun l'ambition de remettre les classes moyennes sur le chemin des bureaux de vote. Mais ces initiatives sont largement limitées au moment des campagnes électorales. Par exemple, la campagne "Choose the Right Councillor», organisé par l'ONG Public Affairs Centre à Bangalore (Jennifer Jalal, "Good Practices in Public Sector Reform : A Few Exemples from Two Indian Cities», in Amita Singh (éd.), Administrative Reforms. Towards Sustainable Practices, Delhi, Sage, 2005, p. 96-116, p. 106), la campagne «Vote Mumbai», organisée par Lok Satta, ou le "Gurgaon Residents Party» créé par People's Action, qui aboutit à la candidature - mais pas à l'élection - d'un représentant des associations de résidents dans cette ville au sud de Delhi. À Chennai, lors des premières élections municipales organisées dans le cadre de la mise en œuvre de la politique de décentralisation, en 1996, plusieurs candidats indépendants, issus des associations de résidents, furent élus, mais l'expérience fut vécue comme un échec pour les élus comme par leurs électeurs, et ne fut pas répétée en 2001 (Pushpa Arabindoo, op. cit., p. 61). 